\title{
Rare Malpositions Following Insertion of the Left Jugular Vein Catheterization: Case Reports and Literature Review

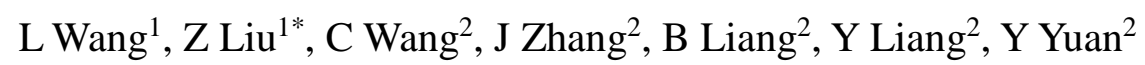

\begin{abstract}
There is a persistent number of patients commencing chronic haemodialysis with tunneled cuffed catheter. Usually, the right internal jugular vein (IJV) is the preferred initial location of consideration for percutaneous insertion. In special situation, catheterization of the left IJV is also a second best in patients with history of multiple access failures. In this report, we present two rare cases of malposition occurring after left IJV catheterization, while following catheter adjustments provide good outcomes with the help of chest radiographs. To our knowledge, the cases exemplify uncommon complications during the left IJV catheterization with few reports in the published literature.
\end{abstract}

Keywords: Catheter malposition, central venous catheter, complications, jugular vein catheterization, left internal

From: ${ }^{1}$ Department of Nephropathy, the First Affiliated Hospital of Zhengzhou University, Zhengzhou 450052, China and ${ }^{2}$ Department of Nephropathy, Zhengzhou Cardiovascular Hospital, Zhengzhou 450016, China.

Correspondence: Dr Z Liu, Department of Nephropathy, The First Affiliated Hospital of Zhengzhou University, No. 1 Jianshe East Road Zhongyuan District, Zhengzhou 450052,China. Tel: +86 37160609557 , Fax: +86 37160609558.

E-mail: zsliucn@126.com 


\section{Complications During the Left IJV Catheterization}

\section{INTRODUCTION}

Central venous catheters (CVC) are commonly utilized to gain vascular access for uremic patients since they offer unique advantages to meet chronic haemodialysis. Various complications of centralvenous catheterization have been reported while the most common complications arise from insertion of the catheter include pneumothorax, misplacement, arterial puncture, local infection, hematomas, and thrombosis (1-4). Malposition of a central venous haemodialysis catheter is an uncommon complication (4\%), requiring appropriate management (5).

Physicians often prefer cannulation of the right internal jugular vein (IJV) to establish central venous access since it provides a more direct path to the superior vena cava, while the left IJV is also a second best in patients with history of multiple access failures. As (Fig. 1) shown, the left IJV catheter during the insertion must negotiate two 90 turns: junction with the subclavian vein and junction with the superior vena cava $(6,7)$.

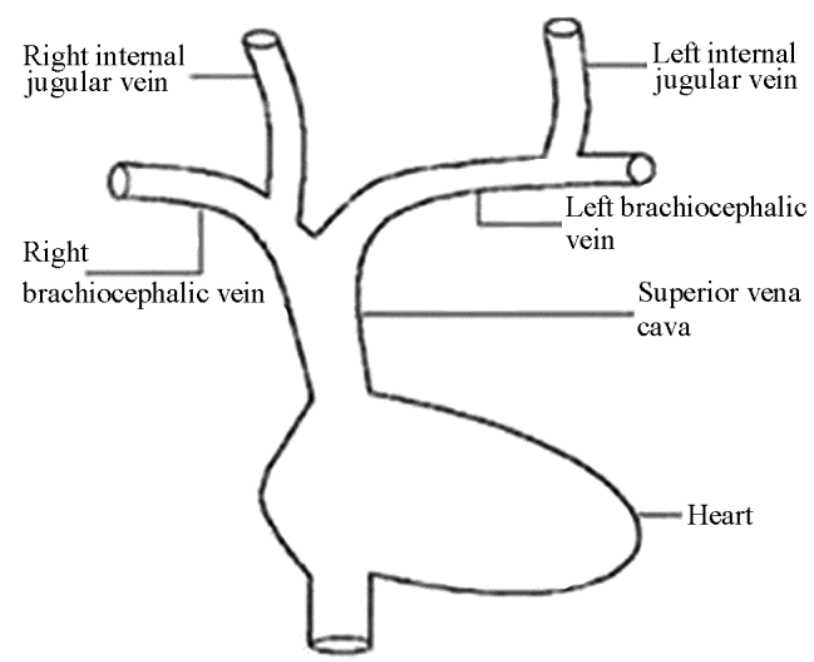

Fig. 1: Anatomic diagram for catheterization at the jugular vein.

It shows that left internal jugular vein catheter negotiate two 90 turns. 


\section{Wang et al}

It is reported that cannulation of the left IJV is more difficult and associated with more malpositions even than the right one $(3,4)$. One reason is that left brachiocephalic vein is anatomically longer and has more smaller tributaries than the right one $(2,3)$. Another reason is that left tributaries anatomically anastomose with the brachiocephalic vein (8). Central venous catheters can be misdirected into smaller tributaries of central veins, particularly when inserting catheter through left central veins.

The report illustrates two rare malposition of central venous catheter in the left IJV catheterization despite the use of fluoroscopy for confirmation of positioning. The main cause of this rare malposition and methods of prevention are discussed below. Although complications we described are uncommon, information on sources of malposition can be extracted to increase the quality of catheterization during the course of insertion in difficult cases.

\section{CASE PRESENTATION}

\section{Patient 1}

A 63-year-old female with end-stage renal disease (ESRD) maintained on haemodialysis since 2009. This study was conducted in accordance with the Declaration of Helsinki. This study was conducted with approval from the Ethics Committee of Zhengzhou University. Written informed consent was obtained from all participants. The patient suffered repeated arteriovenous fistula (AVF) thrombosis. Then a right IJV tunneled cuffed (TC) was created for a long-term dialysis in October 


\section{Complications During the Left IJV Catheterization}

2009. On 11th July 2013, the tunneled cuffed catheter fell out from the percutaneous tunnel asymptomatically. The ultrasonography (Fig. 2A) detected narrowing developed along the right IJV. We attempted to replace a tunneled cuffed catheter to left IJV under the consent of the patient. Under local anaesthesia, a long-term, dual-lumen, cuffed, haemodialysis catheter (BARD, diameter: 14.5Fr, cuff to tip $40 \mathrm{~cm}$ ) was inserted through the left IJV, tunneled subcutaneously at the left prothorax area. Gentle suction was applied from needle, no blood from the venous lumen returned with passage. Then a chest film was evaluated for the presence of malfunction of the catheter. The chest radiography (Fig. 2B) showed that the venous lumen back-folded its route. During next insertion after the catheter's extraction, we chose the site near the former to puncture and insert a tunneled cuffed catheter. We attempted to adjust the direction and depth of the catheter then blood returned satisfactorily during withdrawal of the needle. Another chest film (Fig. 2C) showed the replacement's position after the procedure.

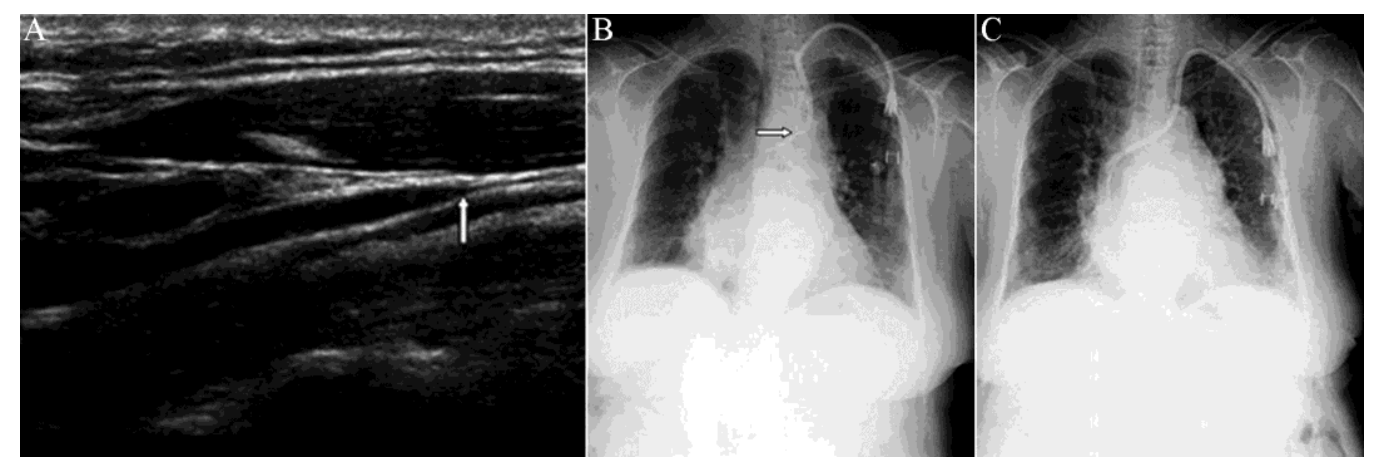

Fig. 2: Graphs showing the adjustment of the catheter resulting from kinking of one lumen in 63-year-old female.

A: The stenotic right jugular vein; the ultrasonography detects that narrowing along the right internal jugular vein; arrow indicates stenosis.

B: Malposition location of a left internal jugular vein catheter; the chest film shows venous lumen reentrant in the vein; Arrow indicates fold.

C: Corrected location of the left internal jugular vein catheter; the chest radiograph shows that luments are at the right position. 


\section{Wang et al}

\section{Patient 2}

A 38-year-old female maintained on haemodialysis for six months, resulting from stage V diabetic nephropathy. This study was conducted in accordance with the Declaration of Helsinki. This study was conducted with approval from the Ethics Committee of Zhengzhou University. Written informed consent was obtained from all participants. One right IJV catheter was created temporarily for dialysis in January 2013. She rejected to exchange for permanent catheter after the AVF failed and her dialysis course turned to be difficult. We had to extract the catheter because of frequent, serious catheter infection. The following ultrasonography (Fig. 3A) detected a thrombus existing in the lumen of IJV after extracting the catheter. A dual-lumen cuffed catheter was placed in left IJV under the former procedure. Aspirated blood through each lumen and little flow of the lumen returned with passage. The chest radiography (Fig. 3B) showed that both of the lumens had lost their traces and turned to the right IJV. After the catheter's extraction, we extended the length of the wire and reintroduced the catheter passing over the wire. Blood returned smoothly during withdrawal of the needle. Another chest film after the procedure (Fig. 3C) showed the replacement position which the catheter was still at malposition. For it worked in good condition during the following-up period, second adjustment did not attempt.

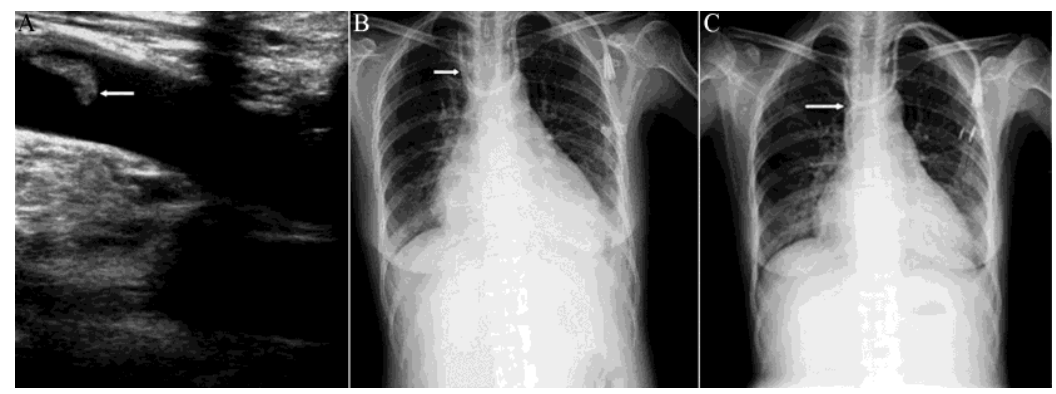

Fig. 3: Graphs showing the adjustment of the catheter resulting from bifurcation of one lumen in 38-year-old female. 


\section{Complications During the Left IJV Catheterization}

A: The thrombotic right jugular vein; it reveals there is thrombus in the lumen of right internal jugular vein. Arrow indicts thrombus.

B: The misplacement of a left internal jugular vein catheter; the chest film shows both of lumens's inadvertent insertion into the right internal jugular vein.

C: Corrected location of the left internal jugular vein catheter; the chest film shows the lift internal jugular vein catheter is still at malposition after replacement.

\section{DISCUSSION}

The left IJV is selected when the right IJV cannulation is unsuccessful or contraindicated for haemodialysis patients with exhaustive AVF. It is not surprising that cannulation of the left IJV is more difficult, and related to more complications than right internal jugular cannulation for the anatomic disadvantage. It is reportedly associated with a higher incidence of complications, which is painful and hazardous to patients, in comparison with the right one (9-12). There are $15 \%$ patients for tunneled CVC with complications and the incidence is greater for left IJV cannulation compared to the right IJV $[20 \%$ vs 10\%] $(11,13,14)$. There are reports of haemodialysis catheter misplacement when insertion was attempted through a vein distally to central vein that was hypoplastic, stenosed, or thrombosed $(4,12-15)$.

Malposition of the catheter is a relatively uncommon complication (4\%) which results in the malfunction of the catheter, requiring appropriate management [catheter's position correction, replacement, or removal] (16-20). This anatomic disadvantage highlights the need for radiographic confirmation of proper catheter 


\section{Wang et al}

location which is tested and verified though our observation.

In the first case, the arterial lumen of the catheter is withdrawn and overlapped in the junction of the left brachiocephalic vein and superior vena cava, which might result from the stenosis or bend of the left brachiocephalic vein. The following adjustments of the site, direction and depth of the puncture needle, provide good outcomes with the help of X-ray examination. In the second case, it is the inadvertent insertion of catheter into the right IJV. Both of lumens of the catheter are directed from the left IJV to the junction of left brachiocephalic vein and superior vena cava and then to contralateral IJV. In the adjustment, the arterial lumen still could not be induced unsatisfactorily, adverse into the right IJV after reinsertion, though the arterial lumen induced properly. It is not causeless that both of the split-tip catheters are aberrantly placed in the vein which results in their dysfunction in our patients.

Tunneled CVCs for dialysis are classified into either split-tipped (dual-lumen) or step-tipped (single-lumen) catheters depending on the catheters tip design.

The variable in design takes an effect on the incidence of complication. The malposition is more likely to occur in the split-tipped catheter through the left IJV because left-sided catheter placement meets serious challenge: longer passage and more bends to the desired location, by contrast to the right one (20-21). Dual-lumen catheters on the left-sided predispose to bifurcate between lumens passing though curved path $(2,5,20)$. It is appeared that the arterial lumen is easier to kink, overlap or reentrant in the vein even step to other vein for lack of wire guidance during the advance of catheter, while venous lumen is not for the guide-wire passing through. 


\section{Complications During the Left IJV Catheterization}

Thus, we consider that the step-tipped catheter is to be less malposition than the split-tipped, although dual-lumen catheter are widely believed to reduce recirculation rates in comparison with to single-lumen one (22). As presented in our report, the tape of the catheter should be taken into account to avoid the lumen's bifurcation or offsetting during advancing catheter. On consideration that dual-lumen catheter is associated with a higher incidence of the divarication throughout the procedure, it indicates that single-lumen would be more applicable to the left-side IJV cannulation.

It is a novel point of view that the step-tipped catheters would be the foremost priority in the left IJV cannulation while split-tipped seems to be more susceptible to failure on base of our observation, which has not been mentioned in the previous literature.

Meanwhile, the guide-wire leads an important role in steering the successful placement of left-sided catheter. If the wire is unexpected to kink, enter into other vein inadvertently or into the vein too short, the catheter would tend to be misplaced or obstructed following the guidance, which presents in the second case.

The nonthrombotic causes of the catheter leading to dysfunctions include the migration, kinking of a wire, or port rotation, which are confirmed by typical imaging findings (4). Chest roentgenograms shortly thereafter reveal whether aberrant positioning of the guide-wire or the catheter has set. As previous reports, it is essential for chest radiograph in detecting anatomic variants and judging the placement of the catheter after the procedure $(4,23)$, which proves to be effective in our study too.

Furthermore, it appears to be feasible that chest roentgenograms or fluoroscopy 


\section{Wang et al}

could also verify correct positioning after advancing the wire, before the insertion of the lumen according to our clinical findings. It is indispensable that making X-ray examination in the midterm of procedure to assess the position of the tip and give guide to the next adjustment after the insertion. That is to say, X-ray examination can be made as a real time image during the course of implantation of the lumen into the vessel.

In the two patients, the analysis of the malposition helps us to have more knowledge for the left-sided catheter placement, maximizing the success of central venous access device placement and minimizing the risk of potential complications, with few cases report in the literature. A potential weakness in our article may be that the number of patients is insufficient to highlight more striking results.

\section{CONCLUSION}

Our results confirm that catheterization is associated with higher failure rates and more complications particularly for left IJV placement. Single-body catheter is the least favourable one with the help of the imaging examination in the insertion. X-ray examination can be made as a real time image before and after the insertion.

\section{CONFLICT OF INTEREST}

All authors have no conflict of interest regarding this paper. 


\section{Complications During the Left IJV Catheterization}

\section{REFERENCES}

1. Gupta P, Guleria S, Sharma S. Mediastinal haematoma: a rare complication following insertion of central venous catheter. Indian J Chest Dis Allied Sci 2011; 53: $225-8$.

2. Skandalos I, Michalopoulos A, Iatrou K, Sombolos K. Hemodialysis catheter malposition induced by a brachiocephalic vein stenosed stent. Ren Failure 2013; 35: $541-3$.

3. Bannon MP, Heller SF, Rivera M. Anatomic considerations for central venous cannulation. Risk Manag Healthc Policy 2011; 4: 27-39.

4. Ahn SJ, Kim HC, Chung JW, An SB, Yin YH, Jae HJ et al. Ultrasound and fluoroscopy-guided placement of central venous ports via internal jugular vein: retrospective analysis of 1254 port implantations at a single center. Korean $\mathbf{J}$ Radiol 2012; 13: 314-23.

5. Skandalos I, Hatzibaloglou A, Evagelou I, Ntitsias T, Samaras A, Visvardis G et al. Deviations of placement / function of permanent central vein catheters for hemodialysis. Int J Artif Organs 2005; 28: 583-90.

6. Ash SR. Advances in tunneled central venous catheters for dialysis: design and performance. Semin Dial 2008; 21: 504-15.

7. Shiao CC, Chu TW, Hung JJ, Hong PJ, Yang CL, Kao JL. An unusual left-sided tunneled catheter in a hemodialysis patient. Ther Apher Dial 2012; 16: 106-7.

8. Sakan S, Basić-Jukić N, Kes P, Stern-Padovan R, Perić M. Malposition of central 


\section{Wang et al}

venous dialysis catheter in the right internal mammary vein in uremic patient: case report. Acta Clin Croat 2011; 50: 623-6.

9. Stolic RV, Stolic DZ. A left jugular vein catheter for hemodialysis malpositioned in right brachiocephalic vein. Iran J Kidney Dis 2012; 6: 98.

10. Moeinipour AA, Amouzeshi A, Joudi M. A rare central venous catheter malposition: a case report. Anesth Pain Med 2014; 4: 16049.

11. Sulek CA, Blas ML, Lobato EB. A randomized study of left versus right internal jugular vein cannulation in adults. J Clin Anesth 2000; 12: 142-5.

12. Mrozek N, Lautrette A, Timsit JF, Souweine B. How to deal with dialysis catheters in the ICU setting. Ann Intensive Care 2012; 2: 48.

13. Yaacob Y, Nguyen DV, Mohamed Z, Ralib AR, Zakaria R, Muda S. Image-guided chemoport insertion by interventional radiologists: a single-center experience on periprocedural complications. Indian J Radiol Imaging 2013; 23: 121-5.

14. Vats HS. Complications of catheters: tunneled and nontunneled. Adv Chronic Kidney Dis 2012; 19: 188-94.

15. Kayashima K, Habe K, Imai K. Left internal jugular venipuncture in real-time ultrasound-guided pediatric central venous cannulation. Masui 2012; 61: 1105-7.

16. Alemohammad M. Central venous catheter insertion problem solving using intravenous catheter: technical communication. Tehran Univ Med J 2013; 70: $724-8$.

17. Shams Vahdati S. Should a double-lumen catheter be withdrawn? J Cardiovasc 


\section{Complications During the Left IJV Catheterization}

Thorac Res 2011; 3: 97-9.

18. Ghatak T, Azim A, Baronia AK, Muzaffar SN. Malposition of central venous catheter in a small tributary of left brachiocephalic vein. Emerg Trauma Shock $2011 ; 4:$ 523-5.

19. Gyaninder PS, Hemanshu P, Bapura KR. Malposition of internal jugular vein catheter into contralateral internal jugular vein: an uncommon position. Indian J Anaesth 2012; 56: 205-7.

20. Tan EK, Tan SG. The permanent catheter. Hemodial Int 2014; 18: 522-4.

21. Chintu MR, Chinnappa S, Bhandari S. Aberrant positioning of a central venous dialysis catheter to reveal a left-sided partial anomalous pulmonary venous connection. Vasc Health Risk Manag 2013; 62: 870-2.

22. Lee HJ, Park SW, Chang IS, Jo YI, Park JH, Lee JH et al. A comparison of standard dual-tip hemodialysis catheter split lumen hemodialysis catheter. Clin Imaging 2013; 37: 251-5.

23. Venugopal AN, Koshy RC, Koshy SM. Role of chest X-ray in citing central venous catheter tip: A few case reports with a brief review of the literature. $\mathrm{J}$ Anaesthesiol Clin Pharmacol 2013; 29: 397-400. 
Wang et al 